\title{
Qualitative Notions of Testability
}

\author{
B. Baumgarten, \\ GMD, TKT \\ Rheinstr. 75 \\ 64295 Darmstadt, Germany \\ baumgart@darmstadt.gmd.de \\ H. Wiland
Karlstr. 9a
68307 Mannheim, Germany \\ H. Wiland
Karlstr. 9a
68307 Mannheim, Germany \\ H. Wiland
Karlstr. 9a
68307 Mannheim, Germany
}

Abstract

Testability, and design for testability, are widely discussed practical issues in software engineering, especially in protocol engineering. Existing definitions (or circumscriptions) of testability seem to be either quite vague, or, if more or less formal, then dedicated only to very special system models. Testability is usually decomposed into aspects like observability and controllability, and these are defined either as qualitative properties or as quantitative measures. We identify a set of qualitative testability properties that we define completely independently from any special system model, only in terms of user-relevant aspects like possible, desired, and undesired system observations or outcomes of experiments.

\section{Keywords}

Testability, Specification theory, Conformance, Semantics

\section{INTRODUCTION}

In theory and practice of testing, testability is often emphasized as a desirable property of specifications and systems. There exist various useful informal discussions of testability $[5,10,18,20,21]$. Related formal notions of observability and controllability were defined in mathematical systems theory for abstract machines $[16,19]$.

In the following we use a general semantic framework for system specification, based on the specification context between systems, system properties and system observations, to discuss general qualitative concepts of testability. We deal only with testability aspects of specifications, not with test implementation matters. 
Moreover we are concentrating on qualitative, rather than quantitative, concepts of testability. The latter could be built upon the former, cf. Section 6 .

The rationale of the chosen framework will be discussed thoroughly in separate papers, e.g. in an investigation of system properties required by specifications [3]. Technically, it draws implicitly on the mathematical properties of Galois schemes [2], based on Galois connections, which have already proved useful in Computer Science $[9,11]$. This paper is part of ASPEKTE, an effort to introduce a theory of specification as a basic common language for specification, verification and testing.

\section{RELATED WORK ON TESTABILITY}

Testability, observability and controllability have been described both formally and informally, and both as qualitative and as quantitative notions.

\section{Informal approaches}

[13] defines testability on the one hand as the degree to which a system or component facilitates the establishment of test criteria and the performance of tests to determine whether those criteria have been met, and on the other hand as the degree to which a requirement is stated in terms that permit establishment of test criteria and performance of tests to determine whether those criteria have been met.

In [20], testability is viewed as the probability that a system will fail on its next execution during testing (with a particular assumed input distribution) if the software includes a fault.

In $[5,8,18,21]$, as in many other sources, testability is circumscribed as the existence of features, properties or characteristics that facilitate the testing process of implementations. Particular aims are to reduce effort or cost and to facilitate the easy application of testing methods and the detection or isolation of existing faults. The testability measure should be a vector of measures of particular testability aspects. [10] identifies observability and controllability as two important factors of testability.

\section{Formal approaches}

Carnap's seminal paper [7] on testability, dating back to 1936, seems to have been quite ignored in Computer Science. Since Kalman's control-theoretical paper [17], controllability and reachability concepts (of states and systems) refer to the possibility of directing a system from given states to other states, while observability concepts refer to the possibility of identifying the unknown state of a system by means of the relationship between certain inputs fed to the system and the outputs obtained from it in a finite time interval.

Early definitions of controllability and observability concepts in the context of abstract machines and automata were proposed by [16] and [19]. [16] defines controllability for Mealy automata as the property that all states can be reached from one another with the appropriate inputs. The automaton is called initial-state determinable if its unknown initial state can be determined by experiments feeding inputs and observing outputs-a generic definition that depends on the kinds of 
experiments permitted. In [19], a non-deterministic Mealy automaton is called observable if a state and an input performed and an output observed in this state determine the next state reached.

In $[8,18]$, controllability and observability are quantified in the setting of finite state machines.

\section{SPECIFICATION CONTEXTS}

\subsection{Definition}

When we deal with specifications, we usually do so within some context, characterized by a population of systems under consideration, the set of their possibly relevant properties, and a set of possible observations of interest. What is practically considered as a system, a property, or an observation in a context depends e.g. on

- the chosen part of reality or field of thinking (e.g. we may be dealing with protocol entities and not with white mice),

- the chosen level of abstraction (e.g. on the level of data base access protocols we may not care about signal wave shapes), and

- the envisaged meaningful uses and abuses of the system (e.g. we may not care about the colour of the inside of the casing of our monitor, even though it certainly has some colour, and we could find it out if we desired to).

In [3], a specification context is defined as a quintuple (Systs, Props, Obs, has_property, permits), where

- Systs is a set of systems,

- Props is a set of properties,

- Obs is a set of observations,

- has_property is a relation between systems and properties, and

- permits is a relation between systems and observations, which, for the purposes of this paper, is non-empty as well as left- and right-total.

In practice, specification contexts will be described by a selected combination of natural language, technical vocabulary and mathematics. In this paper we will not be very much concerned with Props and has_property.

The last item of the above definition concerns three non-degeneracy assumptions dictated by practical considerations. Specification contexts without systems or observations are of little interest. Non-observable systems can be ignored, they are transcendental w.r.t. the given context: we could not (or would not care to) notice such a sys, even if we were standing right in front of it. In order to simplify later definitions, we work only with observations that can really be made on at least one system. Formally, our non-degeneracy assumptions amount to:

Systs $\neq \varnothing \wedge O b s \neq \varnothing$,

$\forall$ sys $\in$ Systs : $\exists$ obs $\in$ Obs : sys permits obs, 
Relationships and transitions between different system contexts, which play an important role in questions of abstraction, refinement and modelling, and for which it sometimes pays to relax (i,ii,iii), will not be investigated here. Instead, we will mostly confine ourselves to an arbitrary single specification context Cont $=($ Systs, Props, Obs, has_property, permits). Definitions are then implicitly referring to Cont. For example, a "behaviour" means a "behaviour in Cont."

\subsection{Observations}

Observations are a central notion in this paper. We explicitly assume that $O b s$ comprises all observations that are both imaginable and of potential interest, in the sense that they may be permitted, desired or undesired. It does not matter whether they are direct or indirect, active or passive, long or short, atomic or complex. As to complexity, for example, on a certain level of specifying a vending machine, both the insertion of a coin and the dispensal of a soft drink may be atomic observations, but we also need more complex observations such as "I inserted the required coin but nothing happened in the next 20 seconds," and "I inserted the required coin and received a drink within 5 seconds." A realizable combination of repeated and/or parallel observations, for example, must also be considered as one complex observation if it is relevant w.r.t. to system conformance.

Later definitions will be implicitly based on the informal assumption that each observation is made in finite time. As the saying goes, "infinity is where things happen that don't." [3] shows that system requirements based on infinite observations may turn out to be void.

\subsection{Behaviours}

Apart from what we know, or assume, about all systems in Systs, whatever we find out about a system, we find out exclusively by means of observations. Every system permits only certain observations. Thus, the (visible) behaviour of a system consists of the observations that can be made of it:

sys_beh: $\left\{\begin{array}{lll}\text { Systs } & \rightarrow & \mathbf{P}(O b s) \\ \text { sys } & \mapsto & \{o b s \in O b s \mid \text { sys permits obs }\}\end{array}\right.$

A behaviour Beh in general can be defined as a set of possible observations, Beh $\subseteq O b s$. At this point, mathematical purists might suggest that a system simply is a behaviour, i.e. a subset of $O b s$. While this would not really change our results, it might be counter-intuitive for many readers; therefore, we stick to the separate set Systs.

Telling two systems apart takes at least one observation possible for one system and impossible for the other, even if it only consists of a name, a serial number or a position in space. Systems sys 1 and sys 2 are indistinguishable if they "behave identically," i.e. if sys_beh(sysl) =sys_beh(sys2), the full abstractness issue of [12]. 


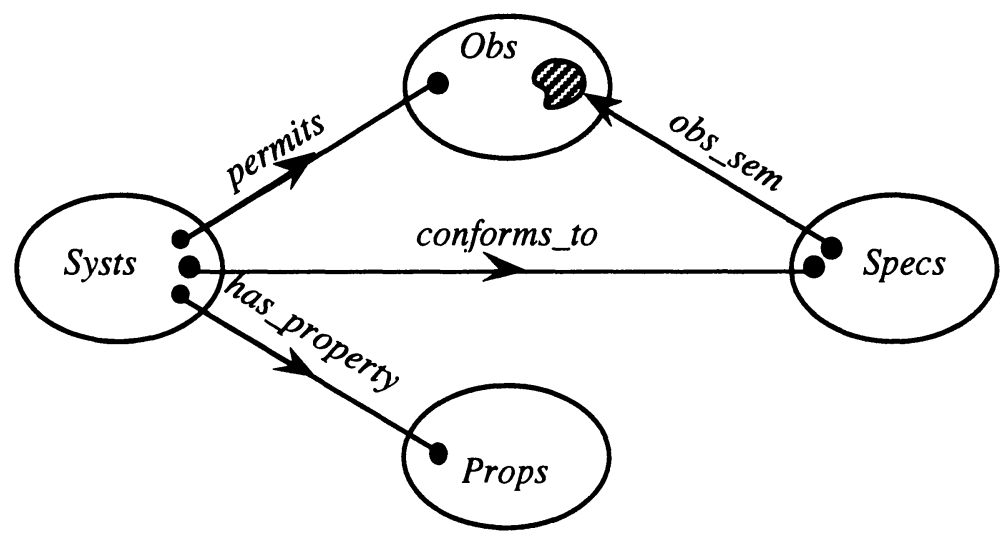

Figure 1: The semantic embedding of specifications into a specification context

\subsection{Specifications and Conformance}

From a user and tester standpoint, the purpose of a specification is to describe the range allowed for the behaviour of the desired systems. Such a behaviour range is - like the behaviour of a single system-also a subset of $O b s$, i.e. a behaviour. If we allow for nondeterminism in systems, the differences between a system behaviour and a specified behaviour range all but vanish. Therefore, we speak uniformly of the "behaviour" both of systems and of specifications.

Systems and specifications defined by means of a formal description technique often comprise internal aspects, like operations on internal variables in programs or invisible steps in transition systems. "Invisible behaviour" often amounts to visible behaviour in a different context permitting other observations, such as program code inspection. Within a given context, invisible aspects of a system are meaningful merely as auxiliary constructs to define (visible) behaviour.

Loosely spoken, a system specification is "anything that defines a behaviour." The latter, i.e. the set of permitted observations, is usually not listed literally, in particular if it is infinite. Rather, it is inferred from the chosen syntactic form of specification, taken from a set Specs of specification terms, via an observational semantics (cf. Figure 1)

obs_sem: Specs $\rightarrow \mathbf{P}(O b s)$.

Two important forms of specification terms are a (finite) list of required properties, such that Specs $\in \mathbf{P}_{\text {fin }}$ (Props), cf. [3], and a term in a formal description language used both for specifications and for abstract systems. In the latter case, Specs = Systs, and obs_sem coincides with sys_beh; this approach can be used nicely in process algebra [12].

Proposing specifications without commitment to Obs or obs_sem may lead to misunderstandings about valid system behaviour and jeopardizes the practical use of specifications. 
A system conforms to a specification (term) spec if its behaviour stays within the range permitted by spec:

sys conforms_to spec $: \Leftrightarrow$ sys_beh(sys) $\subseteq$ obs_sem $($ spec $)$.

Unlike the case of untimed string trace semantics, this definition does not imply that an inactive system conforms to arbitrary specifications, or that a less active system conforms to a more active specification: in practical contexts, temporary inactivity is observable.

We call an observation obs valid for a specification spec if

$\exists$ sys $\in$ Systs: sys conforms_to spec $\wedge$ sys permits obs.

Otherwise, we call it invalid. Any valid observation may have come from a conforming system. Any invalid observation obtained tells us that the investigated system is non-conforming. Note that in pathological cases allowed observations may be invalid (!), as, to take an informal example, when you allow a child to play in the mud, but not to get dirty ...

We call an observation obs validating for a specification spec if

$\forall$ sys $\in$ Systs: sys permits obs $\Rightarrow$ sys conforms_to spec.

Only in very nice contexts do validating observations exist. A non-validating observation is one that may have come from a non-conforming system.

The framework described is fairly general and applies equally well to systems outside of digital information processing. Specializations typical of the latter field of application will be outlined and many of the practical notions about testing-cf. [14] for a representative list - will be formalized within the framework of specification contexts in ASPEKTE papers.

\section{FLAVOURS OF REFUTABILITY AND VALIDATABILITY}

The term "testability" can refer to specifications, systems, and testing environments. In this paper we deal with testability properties as possible properties of specifications. Some questions about specifications that are of obvious interest are

- Can systems be "proven to be conforming" to a specification by testing? Can they be "proven to be non-conforming"?

- Can all systems be proven to be conforming or non-conforming, respectively, or only some, or none?

- Can the diagnosis only be made if the-non-deterministically achieved-test result allows it, so that a decisive observation cannot be enforced?

Or is there a method that guarantees to elicit the decisive observations?

- And if a diagnosis is possible in finite time, can this time be bounded in advance? 
Thus, qualitative aspects of the testability of a specification are whether, through testing, conformance to this specification can be

\section{- validated or refuted}

- for arbitrary or only for some conforming resp. non-conforming systems under observation, or for none of them (strongly or weakly),

- possibly only with luck (non-deterministically, ND) or with a guarantee (deterministically, D),

- in bounded or arbitrary finite time. We will deal with this latter aspect in a separate paper [4] and will merely touch upon it in Section 5.

By "through testing" we mean that even though the specification context may be known, the observer does not know in advance which of the systems she is observing, i.e. which one is the system "under test". Any information about this system's behaviour is collected exclusively by means of observations.

Due to problems with non-determinism and infinite cardinality, we cannot expect to identify the full behaviour of a system by means of observations. Generally, an observation obtained may merely be one among various possible ones, and it does not tell us which other observations were possible. If we can obtain observations repeatedly - say, we can perform some experiment over again, or other experiments, as well - then we may obtain other observations. However, unless our context has nice properties, we will never be sure about the full set of possible observations of the (unknown) system we are observing - or of the full set of possible outcomes of an experiment (cf. 4.2). Even if we actually already obtained all possible observations or outcomes, we would generally not know that we did, i.e. that they make up the full set and that further repetitions will not yield anything new. Note also that we should not expect to obtain information from several observations that cannot be obtained than from any single observation, cf. Section 3.2.

However, an sufficiently "well-behaved" population Systs of systems may permit inferences about system behaviour on the basis of partial information. For instance, if none of the objects in the population ever changes colour, it is sufficient to determine the colour of an object just once in order to know its colour at all times. Advance knowledge about the specification context saves observation work.

We assume that a specification distinguishes between valid and invalid observations not only "in principle" but also in a practical and constructive manner: we do not deal with any "oracle-problem" here. Limits imposed by computability and complexity problems deserve a separate treatment.

A specification spec is called (contextually) contradictory if it does not allow a single observation (nor any system, due to (ii)). A specification spec is called (contextually) void if it allows all observations, obs_sem $(\mathrm{spec})=O b s$, such that every system conforms. [3] treats non-trivial aspects of voidness. In practical situations, such pathological specifications should not arise.

The observational framework developed above permits us to formalize at least "non-deterministic" refutability and validatability, more precisely: without recourse 
to notions of (non)determinism. It is only in addressing determinism that we have to distinguish between observer and system behaviour, as we do in Section 4.2.

\subsection{If it may take luck ...}

Let spec be a non-void specification. Our definitions entail that the observation of any non-conforming system can reveal its non-conformance, though possibly only with luck, namely if we make one of the observations that the system permits and which are not valid by way of spec. Non-determinism lies in the possibility that the relevant invalid obs merely may, but need not necessarily, turn up.

\section{THEOREM 1 [REFUTABILITY]:}

Every non-void specification spec is ND-refutable in the following sense:

$\exists$ Evidence $\subseteq$ Obs: Evidence $\neq \varnothing \wedge \forall$ sys $\in$ Systs:

( $\forall$ obs $\in$ Evidence: sys permits obs $\Rightarrow \neg$ sys conforms_to spec)

$\wedge(\neg$ sys conforms_to spec $\Rightarrow$ Jobs $\in$ Evidence: sys permits obs $)$.

Proof: Take $O b s \backslash o b s \_s e m(s p e c)$ as Evidence .

While everything is quite trivial as far as refutation is concerned, things are slightly more complicated if it comes to validation. There, we can distinguish between the possibilities to validate conformance of some or of all conforming systems by means of observation. We will use "non-deterministically" not as the contrary of "deterministically" but rather as the more general notion, of which the latter is but a special case.

Let spec be a non-contradictory specification. In agreement with well-known limits to the power of testing, we will see that our definitions do not entail that the observation of a conforming system can generally reveal its conformance, not even with a streak of luck in the observations obtained. In some rare contexts and for some specifications, however, conformance can really be validated by means of observation. We refrain from defining validatability or refutability conditions for systems (with respect to specifications), because in the testing situation the system under test, in particular its full behaviour, is usually unknown.

A non-contradictory specification spec is weakly ND-validatable if some conforming system can actually be shown to be conforming, though possibly only by luck. By non-degeneracy arguments this amounts to saying that there exists an observation that can only be obtained from systems that conform to spec, i.e.

$\exists$ obs $\in$ Obs: $\forall$ sys $\in$ Systs: sys permits obs $\Rightarrow$ sys conforms_to spec.

In order to facilitate the comparison of this formula with the one in the next definition we note in passing that it is equivalent to

$\exists$ Evidence $\subseteq$ Obs: Evidence $\neq \varnothing \wedge$

$\forall$ sys $\in$ Systs, obs $\in$ Evidence: sys permits obs $\Rightarrow$ sys conforms_to spec. 
Let us discuss why these formulas reflect our intuitively formulated idea. We find out about conformance by some evidence in the form of a suitable, possibly complex, tell-tale observation. We can be sure to have found out about conformance, if we can infer from this observation that the observed (unknown) system is conforming. All we know about the system is that it permits the observation we made; apart from that it could be any member of Systs. Therefore, any system permitting this observation must be conforming, if we want to be certain of our diagnosis. Conversely, if the formula holds and a tell-tale obs turns up, then the system we have just observed must be conforming.

In Figure 2, specl is not even weakly ND-validatable: every observation may have come from the non-conforming system $b$.

A non-contradictory specification spec is strongly ND-validatable if any given conforming system can actually be shown to be conforming, at least by luck. This amounts to the existence of a set of observations that can only be obtained from systems conforming to spec and such that each conforming system permits at least one observation in this set, i.e.

\section{$\exists$ Evidence $\subseteq$ Obs: Evidence $\neq \varnothing \wedge \forall$ sys $\in$ Systs:}

( $\forall$ obs $\in$ Evidence: sys permits obs $\Rightarrow$ sys conforms_to spec)

$\wedge$ (sys conforms_to spec $\Rightarrow \exists$ obs $\in$ Evidence: sys permits obs $)$.

Non-emptiness could be dropped, because it can be derived.

Again: why does this formula reflect the intuitively formulated idea?-We find out about conformance by some tell-tale observation obs. All tell-tale observations form a set Evidence. Permitting an observation in evidence must again be reserved for conforming systems, otherwise this would not indicate conformance reliably. Hence the second line. Now we want this validation to be possible for every conforming system. Thus, every one of them must permit some the tell-tale observations in Evidence.

Due to space limitations, we confine ourselves to the above two discussions of the agreement between intuition and formal definition. Readers are invited to analyse in a similar manner each of the notions defined in the remainder of this text.

\section{THEOREM 2 [STRONG IMPLIES WEAK]:}

Strong ND-validatability implies weak ND-validatability.

The proof is another simple exercise, if we note that Evidence above must be non-empty.

From a practical point of view, different as these two notions of validatability look, they do have a similar effect on testing: testing a conforming system against a (weakly or strongly) ND-validatable specification may, but need not, prove that the system conforms. When a conforming system is not identified as conforming by observation, then it does not matter too much whether that happened because it was one of the systems for which no telltale observation exists, or because the system happened this time not to yield a-principally possible-telltale observation. 

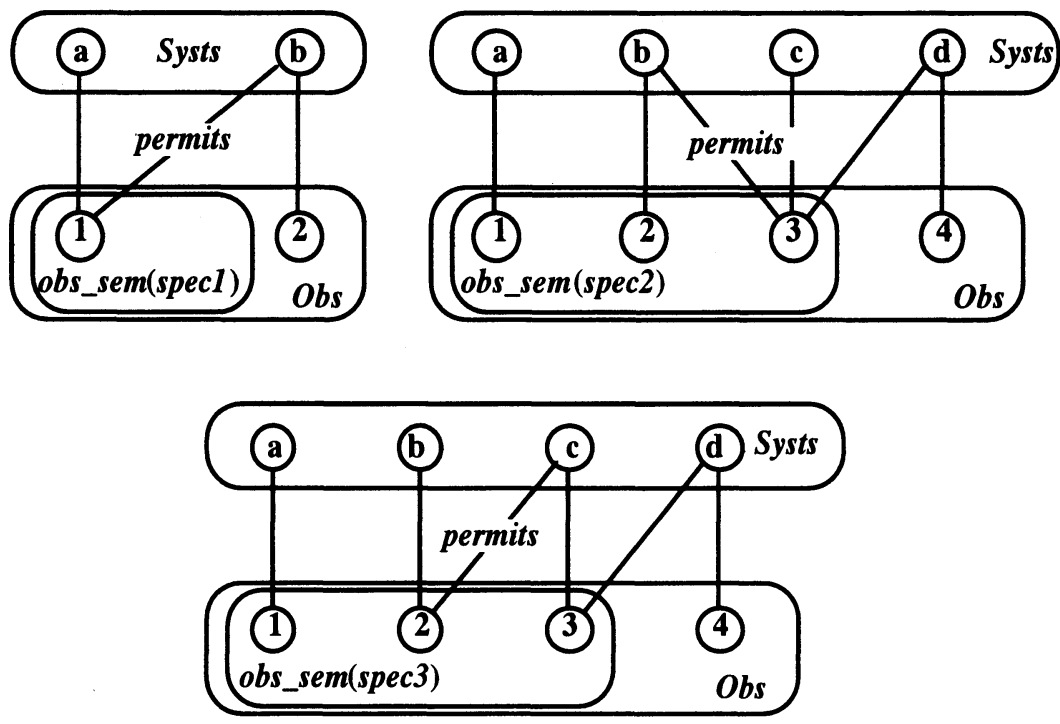

Figure 2: Three specifications in their specification contexts

In Figure 2, spec2 is weakly but not strongly ND-validatable: system $a$ can be validated (shown conforming) by observation $1, b$ can be validated by 2 , but not by 3; $c$ cannot be validated at all. spec 3 is strongly ND-validatable: $a$ can be validated by 1 , both $b$ and $c$ can be validated by 2 , though $c$ possibly only with luck.

\subsection{Experimental specification contexts}

Of course a tester's work is much alleviated if it does not take luck to discover possible invalid behaviour. It is desirable to have methods to arrive with certainty at certain results, be they confirmations (with which we do not deal here ${ }^{1}$ ), refutations or validations. In order to treat the enforceability of refutations or validations formally, we introduce a slight refinement in our framework. We distinguish between a behaviour part determined by the observer, called experiments, and possible outcomes of their interaction with the observed system. Non-determinism w.r.t. the outcome may creep in both by random elements in the experiment and by nondeterminism in the system behaviour.

An experimental specification context is a specification context ExpCont $=$ (Systs, Props, Obs, has_property, permits) (hence fulfilling (i,ii,iii)), where Obs is a left-total relation between a set Exps of experiments and a set Outs of outcomes, and thus a subset of Exps $\times$ Outs. This structure is related with the observation frameworks of [6] and even closer with the observation schemes of [1]. For example, both Systs and Exps may be a set of timed automata, while Outs may consist of the timed traces of their common transitions.

1) A confirmation is basically a non-refuting observation, but often linked with probabilistic aspects that are topics of ongoing research in the philosophy of science. 
The possibility that the experiment exp performed on system sys yields the outcome out, sys permits (exp, out), is also written as (sys,exp) may_yield out, while poss_outs(sys,exp) $:=\{$ out $\in$ Outs $\mid$ (sys,exp) may_yield out $\}$ denotes the set of possible outcomes of exp performed on system sys. By requiring Obs to be lefttotal, we postulate from an experimental specification context that every experiment performed on a system yields at least one outcome,

$\forall$ sys $\in$ Systs, exp $\in$ Exps: poss_outs(sys,exp $) \neq \varnothing$.

This means practically that we never wait eternally for an experiment to end (how could we, anyway?), and that we treat test log entries like "nothing happened, so we broke off after one hour" or "test equipment could not be connected to the system under test" as possible outcomes. Note also that an experiment may comprise a fullfledged hierarchy of sub-experiments, with intermediate decisions based on the part of the outcome observed so far.

Similarly as for observations, any performable combination of experiments is considered as another experiment, such that we cannot obtain from a performable combination of experiments more information than from a single experiment.

Similarly as various observations can be obtained from a system, a given experiment performed on a given system may yield various outcomes. By the same arguments as at the beginning of Section 4, even if all outcomes that may be yielded by a fixed unknown system and a known experiment have actually occurred and have been registered, this fact will generally remain unnoticed by the observer.

The definition of a specification carries over without change such that a specification defines which outcomes are allowed for which experiments. We call an outcome out valid for a specification spec and an experiment exp, if (exp,out) is a valid observation. Otherwise, we call it invalid. Valid and invalid outcomes, respectively, form the sets valid(spec,exp) and invalid(spec,exp). We call an outcome out validating for a specification spec and an experiment exp, if (exp,out) is a validating observation.

By simple application of our previous testability definitions we obtain ...

THEOREM 3 [EXPERIMENTAL ND-REFUTABILITY AND ND-VALIDATABILITY]: In the experimental specification context ExpCont,

- any non-void specification spec is ND-refutable in the sense that $\exists \exp \in \operatorname{Exp}$, out $\in$ Outs: $\forall$ sys $\in$ Systs:

(sys,exp) may_yield out $\Rightarrow \neg$ sys conforms_to spec;

- a non-contradictory specification spec is weakly ND-validatable iff $\exists \exp \in$ Exps, out $\in$ Outs: $\forall$ sys $\in$ Systs:

(sys,exp) may_yield out $\Rightarrow$ sys conforms_to spec;

- a non-contradictory specification spec is strongly ND-validatable iff $\exists$ Evidence $\subseteq$ Exps $\times$ Outs $: \forall$ sys $\in$ Systs:

$(\forall($ exp, out $) \in$ Evidence: $($ sys, exp $)$ may_yield out $\Rightarrow$ sys conforms_to spec $)$ $\wedge($ sys conforms_to spec $\Rightarrow \exists($ exp, out $) \in$ Evidence: $($ sys,exp $)$ may_yield out $)$. 


\subsection{If it shall not take luck ...}

A non-void specification spec is weakly D-refutable if there exists an experiment by which some non-conforming system will definitely reveal that it is nonconforming, again even if the observer does not know which particular system "under test" she is observing. This is the case iff there exist at least one non-conforming system sys 0 and one experiment exp such that performing exp on sys 0 yields nothing but invalid outcomes, or formally:

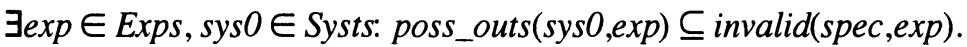

Informally spoken, membership to invalid(spec,exp) is assumed to be effectively decidable. Oracle problems are not considered in this paper.
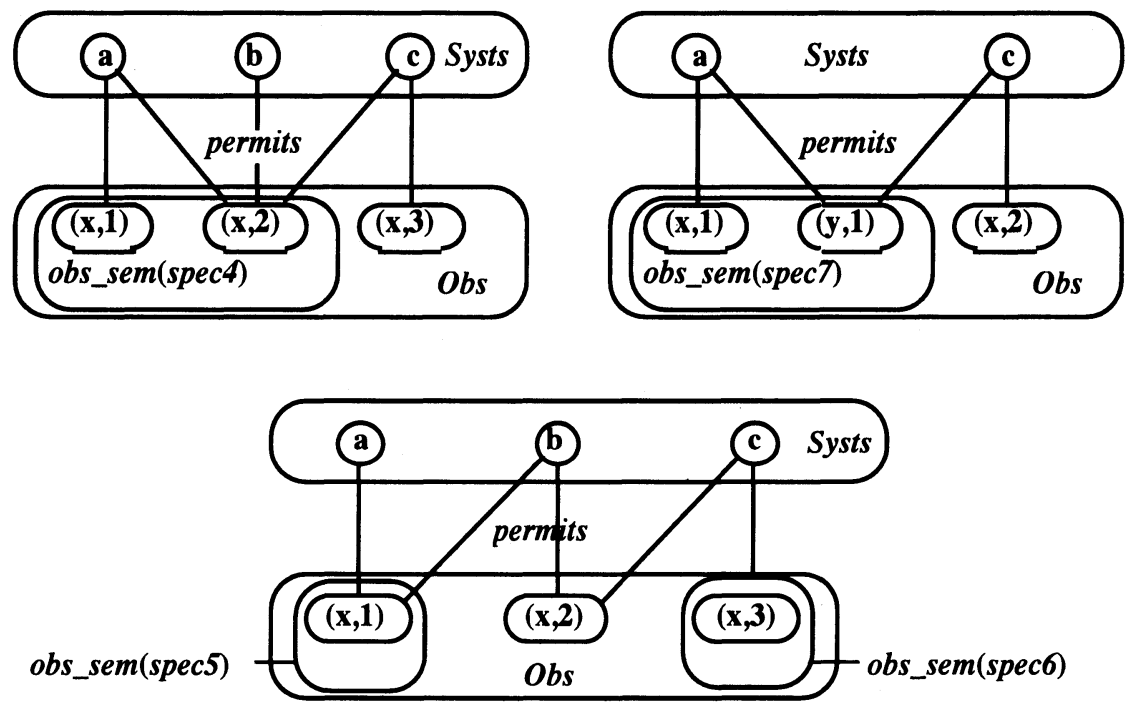

Figure 3: Four specifications in their experimental specification contexts

spec4 in Figure 3 is not even weakly D-refutable. There is only one possible experiment, $x$. No experiment, performed on an unknown system, guarantees to reveal non-conformance (if any) on the basis of each possible outcome. While outcome 3 would indeed reveal non-conformance, each experiment with each valid system may equally well produce the outcome 2 , which may have come from the invalid system $c$.

A non-void specification spec is strongly D-refutable if there exists an experiment by which any non-conforming-possibly unknown-system will definitely reveal that it is non-conforming. This is the case iff there exists at least one experiment exp that-performed on any non-conforming system sys-can only yield invalid outcomes, or formally: 
$\exists \exp \in$ Exps: $\forall$ sys $\in$ Systs:

$\neg$ sys conforms_to spec $\Rightarrow$ poss_outs $($ sys,exp $) \subseteq$ invalid(spec,exp).

In Figure 3, spec5 is weakly but not strongly D-refutable; we will always observe non-conformance while experimenting with $c$, but need luck to detect non-conformance if $b$ is submitted to experiments. spec 7 is strongly D-refutable and demonstrates that generally the right experiment must be chosen: only $x$ will reveal nonconformance.

A non-contradictory specification spec is weakly $\mathbf{D}$-validatable if there exists an experiment by which some possibly unknown conforming system can be forced to reveal that it is conforming. This is the case iff there exists at least one experiment exp and one conforming system sys 0 such that exp performed on sys 0 can only yield outcomes that can only be obtained from a conforming system, or formally:

$\exists \exp \in \operatorname{Exps,}$ sys $0 \in$ Systs: poss_outs(sys $0, \exp ) \subseteq$ validating(spec,exp).

In Figure 3, spec5 is not weakly D-validatable; spec6 is weakly D-validatable but not weakly D-refutable.

A non-contradictory specification spec is strongly D-validatable if there exists an experiment by which any possibly unknown conforming system can be forced to reveal that it is conforming. This is the case iff there exists at least one experiment exp that-performed on any conforming system sys-can only yield validating outcomes, or formally:

$\exists \exp \in$ Exps: $\forall$ sys $\in$ Systs:

sys conforms_to spec $\Rightarrow$ poss_outs(sys,exp) $\subseteq$ validating(spec,exp).

In Figure 3, spec6 is weakly D-validatable but not strongly D-validatable. spec7 is strongly D-validatable and demonstrates that generally the right experiment must be chosen.

The fact that the specification spec7 in Figure 3 is both strongly D-refutable and strongly D-validatable is not coincidental:

THEOREM 4 [EQUIV. OF STRONG D-REFUTABILITY AND -VALIDATABILITY]: In an experimental specification context, a non-void and non-contradictory specification is strongly D-refutable iff it is strongly D-validatable.

Proof: Let spec be strongly D-refutable and exp as in the corresponding definition. If a system sys conforms to $s p e c$, then exp can yield with sys only outcomes in valid(spec,exp). Thus each outcome is outside of invalid(spec,exp) and is, by strong refutability, only possible for conforming systems, hence validating.

The other direction, from strong D-validatability to strong D-refutability runs analogously.

\section{THEOREM 5 [STRONG IMPLIES WEAK]:}

For a non-void and non-contradictory specification, strong D-validatability implies weak D-validatability, and strong D-refutability implies weak D-refutability.

The proof is trivial. 


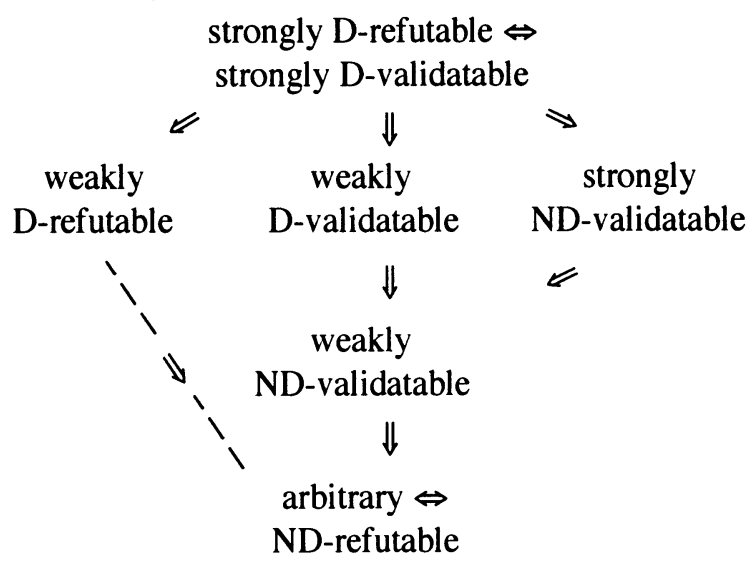

Figure 4: Implications between testability properties

THEOREM 6 [D IMPLIES ND]:

For a non-void and non-contradictory specification, strong D-validatability implies strong ND-validatability, and weak D-validatability implies weak ND-validatability.

Proof: For the "strong" property, take Evidence := $\{\exp \} \times$ validating $(s p e c, \exp )$; the weak part is trivial.

Summarizing the dependencies found so far for non-degenerate specifications, we obtain the implications depicted in Figure 4.

\section{TIME, BOUNDED AND UNBOUNDED}

Up to this point, we have not dealt with questions of duration or complexity of observations, experiments or outcomes. It is certainly of practical interest for all parties involved, be they customers or contractors, implementors, users or testers, to assure that each observation or outcome is obtained in a finite time interval. This is particularly the case in commercial testing, where only a previously fixed period of time is available for the performance of test cases (experiments) and test suites (sets of experiments). Breaking off a test amounts to the performance of another, shorter, test. At any rate, an observation or outcome only achieved after an infinite time span is not achieved at all, or at least not in this world.

An interesting distinction is whether for every experiment there is a pre-defined time limit within which it leads to an outcome, or whether there is no such limit. The latter is possible, even though each experiment performed on any system leads to an outcome in finite time: imagine that there are infinitely many systems sys $s_{1}$, sys $_{2}, \ldots$ and that it takes $n$ seconds for the outcome to appear if the experiment exp is performed on system $s y s_{n}$. However, temporally open-ended testing, if it is undertaken at all, will usually be performed on the basis of payment per time unit. 
Nobody would risk to work for payment only after delivery for an effort that may possibly not be completed during one's lifetime.

Due to the multitude of new notions arising in this context, this topic is treated in a separate paper [4].

\section{CONCLUSION AND OUTLOOK}

General remarks about some poorly defined phenomenon called testability are prone to ambiguity, if not even meaningless. The purpose of this paper was to define clearly various intuitive qualitative aspects of testability and to distinguish carefully among them.

Mathematically, our definitions could be formulated more elegantly in terms of coand contravariant Galois connections, cf. [2,3]. But we did not want to presume foreknowledge in this area.

The testability properties identified in this paper do not relate very obviously. with the legacy concepts outlined in Section 2, except with those in [7]. If we consider as our population of systems one given Mealy automaton in various start states, input sequences as experiments and output sequences as outcomes, and use the claims of a particular start state as specifications, then the initial-state determinability of [16] can be identified with the strong D-validatability of all specifications. It should be interesting to find out the "specification contexts" behind other testability definitions, i.e. which systems, observations and specifications were meant. Controllability in [16], and similarly observability in [19], seem to be mainly properties of all systems, or of Systs, rather than of single specifications: with their aid more specifications become testable, i.e. refutable or validatable in some of the senses introduced in this paper. Thus, testability has definitely even more flavours than those introduced in the present paper, no matter how obvious (and unfortunately numerous) they are.

It should be interesting to find clear testability notions not only for specifications, but also for system properties in Props, required in or derived from specifications. This could lead to a clear definition of the slightly vague concept of test purpose. Similarly, we would like to spend future efforts on relating FMCT notions [15]

As mentioned in the text, the oracle problem, with its aspects of computability, decidability and complexity, imposes limits to effective refutation and validation and therefore deserves closer investigation.

Future efforts should be spent on quantifying testability notions defined in this paper. Experiments and observations can be weighted by cost functions, such that minimum or maximum costs for refutation or validation may be computed. Moreover, if probability distributions are available for non-deterministic alternatives, then expected return-for-investment relationships might be computed for various testing strategies. These distributions may concern the probability of various systems to be submitted to testing against a given spec, or the probabilities of certain observations being made or outcomes turning up in an experiment.

The authors are indebted to Boris Beizer and Olaf Henniger for fruitful discussions. 


\section{REFERENCES}

[1] B. Baumgarten: Die algebraische Spezifikation von Prozessen. Course notes, TH Darmstadt, WS 1992/93

[2] B. Baumgarten: Mathematical Aspects of Specification Theory. Arbeitspapiere der GMD Nr. 1066, 1997

[3] B. Baumgarten: The Observational Significance of System Requirements. Submitted for publication, 1998

[4] B. Baumgarten, O. Henniger: Testability with Unbounded Testing Strategies. Submitted for publication, 1998

[5] B. Beizer: Software Testing Techniques. 2nd ed., V. Nostrand Reinhold, 1990

[6] E. Brinksma et al: A Formal Approach to Conformance Testing. In: Protocol Test Systems, J. de Meer, L. Mackert, W. Effelsberg (eds.), Elsevier/NorthHolland, pp 349-363, 1990

[7] R. Carnap: Testability and Meaning. Philosophy of Science, 3/4, pp 420-471, 4/1, pp 1-40, 1936/37

[8] R. Dssouli et al.: Towards testable communication software. In: Protocol Test Systems VIII, A. Cavalli, S. Budkowski (eds.), Chapman\&Hall, pp 237-251, 1996

[9] J.L. Fiadeiro, J.F.Costa: Mirror, Mirror in my Hand: a duality between specifications and models of process behaviour. Mathematical Structures in Computer Science 6/4, pp. 353-373, 1996

[10] R.S. Friedman: Testability of Software Components. IEEE Trans. on Software Engineering, 17/6, pp 553-564, 1991

[11] B. Ganter, R. Wille: Formal Concept Analysis. Springer, 1998

[12] M. Hennessy: Algebraic theory of processes. MIT-Press, 1988

[13] IEEE Standard Glossary of Software Engineering Terminology, 1990

[14] ISO/IEC IS 9646-1: Conformance Testing Methodology and Framework, Part 1: General Concepts, 2nd ed., 1994

[15] ISO/IEC DIS 13245/ ITU-T Z.500: Formal Methods in Conformance Testing, 1997

[16] R.E Kalman, P.L. Falb, M.A. Arbib: Topics in Mathematical System Theory. McGraw-Hill, 1969

[17] R.E Kalman: On the General Theory of Control Systems. Proc. Ist Intl. Congr. on Automatic Control, Butterworth, pp 481-492, 1961

[18] A. Petrenko, R. Dssouli, H. König: On Evaluation of Testability of Protocol Structures. In: Protocol Test Systems VI, O. Rafiq (ed.), Chapman\&Hall, pp 111-123, 1994

[19] P.H. Starke: Abstrakte Automaten. Deutscher Verlag der Wissenschaften, 1969

[20] J.M. Voas, K.W. Miller: Software Testability: The New Verification. IEEE Software, May 1995

[21] S.T. Vuong, A.A.F. Loureiro, S.T. Chanson: A Framework for the Design for Testability of Communication Protocols. In: Protocol Test Systems VI, O. Rafiq (ed.), Chapman\&Hall, pp 89-108, 1994 